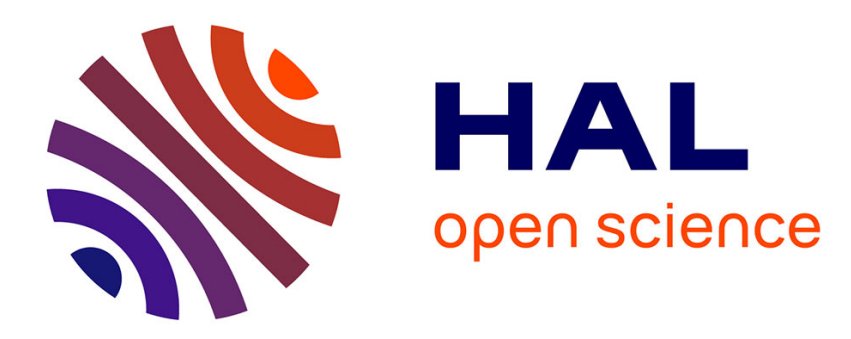

\title{
Mécanisme de croissance et formation des défauts dans les couches minces en microscopie et diffraction électroniques
}

\author{
Michel Froment
}

\section{- To cite this version:}

Michel Froment. Mécanisme de croissance et formation des défauts dans les couches minces en microscopie et diffraction électroniques. Revue de Physique Appliquée, 1980, 15 (2), pp.271-276. 10.1051/rphysap:01980001502027100 . jpa-00244725

HAL Id: jpa-00244725

https://hal.science/jpa-00244725

Submitted on 1 Jan 1980

HAL is a multi-disciplinary open access archive for the deposit and dissemination of scientific research documents, whether they are published or not. The documents may come from teaching and research institutions in France or abroad, or from public or private research centers.
L'archive ouverte pluridisciplinaire $\mathbf{H A L}$, est destinée au dépôt et à la diffusion de documents scientifiques de niveau recherche, publiés ou non, émanant des établissements d'enseignement et de recherche français ou étrangers, des laboratoires publics ou privés. 


\title{
Mécanisme de croissance et formation des défauts dans les couches minces en microscopie et diffraction électroniques
}

\author{
M. Froment (*) \\ Groupe de Recherche n" 4 du C.N.R.S. Physique des Liquides et Electrochimie, associé à l'Université Pierre-et-Marie-Curie, \\ 4, place Jussieu, 75230 Paris Cedex 05, France
}

\begin{abstract}
Résumé. - On étudie la formation des dislocations, défauts d'empilements, parois de double positionnement, macles... en se rattachant au mécanisme de nucléation et de croissance. On examine plus particulièrement le cas des dislocations interfaciales lors de la croissance couche par couche. Dans le cas de la nucléation tridimensionnelle, on étudie les défauts formés par la coalescence des îlots. La nucléation d'agrégats paracristallins se transformant en cristallites multimaclés est aussi considérée. On montre que ces arrangements de macles sont très importants pour la formation de couches à structures dendritique ou texturée.
\end{abstract}

\begin{abstract}
The formation of dislocations, stacking faults, double positionning boundaries, twins... is studied according the mechanism of nucleation and growth. Misfit dislocations appearing during layer by layer growth are specially examined. In the case of three-dimensional nucleation the formation of defects during the coalescence of islands is described. The nucleation of paracrystallite clusters which transform during the growth into multi twinned particles is also considered. These twins arrangements are very important for the formation of dendritic and textured films.
\end{abstract}

1. Introduction. - Lorsqu'on examine la très vaste bibliographie consacrée aux couches minces, on ne peut que constater le rôle essentiel joué par les techniques de microscopie et de diffraction électroniques dans la compréhension des nombreux phénomènes liés à la formation de ces couches : nucléation, croissance, épitaxie, apparition de défauts, rôle de ces défauts dans le mécanisme de croissance.... En outre, et moyennant une adaptation des techniques de préparation des objets, la microscopie électronique permet l'examen de matériaux en couches minces de nature très différente (métaux, semi-conducteurs, composés ioniques ou covalents...) et obtenus selon des procédés extrêmement variés (dépôts à partir de la phase vapeur, de solutions aqueuses, de sels fondus, par électrocristallisation...). C'est dire la richesse des exemples dont on dispose pour illustrer les possibilités de la microscopie électronique mais aussi le risque de tomber dans la présentation d'un album d'images. Il nous a semblé plus intéressant, quitte à simplifier les phénomènes, de montrer l'apport de la microscopie électronique pour l'étude des défauts cristallins en se rattachant à un certain nombre d'étapes ou de mécanismes importants de la croissance des couches minces.

$\left(^{*}\right)$ Conférence présentée au Congrès de la Société Française de Physique (Toulouse)
Parmi les nombreux défauts susceptibles d'apparaître, nous nous sommes limités aux dislocations et aux défauts plans tels que les défauts d'empilement et les macles, compte tenu du rôle important que jouent ces défauts dans des stades ultérieurs de la croissance et dans les propriétés des couches minces; les joints de grains, et certains autres défauts (lacunes, antiphases, ...) sont traités dans d'autres exposés.

\section{Défauts cristallins et mécanismes de croissance. -} Bien que les développements récents des théories de la croissance cristalline soulignent la difficulté de séparer les stades de nucléation et de croissance, et l'intérêt de ne considérer qu'une théorie unique de nucléation s'adaptant à tous les cas, il est assez commode d'aborder la formation des défauts cristallins en fonction du mode de croissance qui leur a donné naissance. Le mécanisme de nucléation et de croissance ultérieure dépend étroitement des interactions entre le dépôt et le support. On a classé les modes de croissance en considérant les énergies superficielles macroscopiques [1] ou les énergies de liaison entre deux atomes du dépôt $\left(\psi_{\mathrm{AA}}\right)$ et entre un atome du dépôt et du support $\left(\psi_{\mathrm{AB}}\right)$. Si $\psi_{\mathrm{AB}}<\psi_{\mathrm{AA}}$, on a une croissance par îlots tridimensionnels (mécanisme de Volmer-Weber); si $\psi_{\mathrm{AB}}>\psi_{\mathrm{AA}}$ la croissance s'effectue couche par couche (mécanisme de Frank van der Merwe) enfin si 
$\psi_{\mathrm{AB}} \approx \psi_{\mathrm{AA}}$, la croissance est tridimensionnelle sur une monocouche adsorbée (mécanisme de Stranski-Krastanov). Nous traiterons ici des défauts formés lors des 2 premiers modes de croissance. Le mécanisme de Stranski-Krastanov, observé lors de la condensation de métaux (Ag, $\mathrm{Au}$ ) sur des semiconducteurs $(\mathrm{Ge}, \mathrm{Si})$ conduit en effet à des situations compliquées en ce qui concerne la structure et les propriétés de la première couche adsorbée [2]. Il faut souligner que le passage d'un mode de croissance à l'autre peut être observé avec un même système support-dépôt, à la suite d'une modification des conditions régnant à l'interface. Ainsi les phénomènes d'adsorption d'atomes ou de molécules étrangères aux interfaces métalgaz ou métal-électrolyte peuvent conduire d'une croissance couche par couche à une croissance par îlots tridimensionnels [3]. On trouvera dans [4] une analyse détaillée des principales méthodes physiques permettant d'identifier ces différents modes de croissance, en particulier dans le cas de la phase vapeur. En électrocristallisation, il est avantageux d'utiliser des méthodes cinétiques [5].

3. Dislocations. - L'apparition de dislocations au cours de la croissance de couches minces peut avoir différentes causes : continuation des dislocations présentes dans le support, dislocations interfaciales dues à l'accommodation des réseaux du dépôt et du support, dislocations liées à la coalescence d'îlots, formation de boucles de dislocations après rassemblement de défauts ponctuels... La continuation des dislocations présentes dans le support est liée à un mécanisme de croissance couche par couche favorisant une croissance épitaxique. Si certaines des dislocations du support ont un vecteur de Burgers avec une composante normale à l'interface, il en résulte l'existence d'une marche dans le support et la possibilité d'une croissance accélérée ne nécessitant pas de nucléation bidimensionnelle. Cet effet a été particulièrement étudié dans le cas de l'électrocristallisation de l'argent où la mise en œuvre de monocristaux exempts de toute dislocation émergeant à l'interface a permis de mettre en évidence une surtension de nucléation et d'étudier quantitativement la nucléation bidimensionnelle [5]. L'existence de dislocations vis émergeant à l'interface entraîne la formation de pyramides et les irrégularités superficielles ainsi créées peuvent jouer le rôle de sites de nucléation; les germes ainsi formés n'ont plus forcément de relation d'orientation avec le support et il en résulte une détérioration de la croissance épitaxique [6, 7].

Dans le cas d'une croissance par îlots, il y a de nombreuses possibilités de formation de dislocations au moment de la coalescence. Ces phénomènes ont été étudiés grâce à des observations in situ en microscopie électronique à transmission (or, argent et plomb sur $\mathrm{MoS}_{2}$, argent sur $\mathrm{MgO}$ ) [8].

Dans ces systèmes, il se forme des franges de moiré distantes de 1,5 à $5 \mathrm{~nm}$ qui facilitent la mise en évidence de défauts cristallins. On observe en général que les germes n'ont pas de défauts au départ et que les dislocations apparaissent lors de l'accommodation de petits déplacements de translation et de rotation de 2 germes en cours de coalescence; ces mouvements sont rendus possibles par la mobilité des germes montrée en particulier par R. Kern et ses collaborateurs [9]. Tant que les germes coalescés restent petits, les dislocations peuvent s'éliminer. Lorsque leur taille devient plus importante, la migration des dislocations est plus difficile et la densité de dislocations augmente.

Des parois de dislocations coins d'organisation très voisine de celles des joints de faible désorientation peuvent également se former pour accommoder 2 germes tournés l'un par rapport à l'autre.

On insistera ici sur la formation des dislocations interfaciales qui illustrent bien les possibilités de la microscopie et de la diffraction électroniques. Ces dislocations apparaissent lors de la croissance épitaxique ce qui implique que certains plans ou rangées cristallines sont communes aux deux réseaux en contact à l'interface et que le désaccord paramétrique n'est pas trop important. Dans le modèle proposé dès 1949 par Frank et Van der Merwe [10], on suppose une croissance couche par couche s'effectuant en deux étapes. Dans la première, il y a adaptation totale du réseau du dépôt à celui du support (pseudomorphisme), malgré la différence paramétrique

$$
f=\frac{a-b}{a} \text {. }
$$

Cependant le dépôt est fortement contraint et à une épaisseur critique $h_{\mathrm{c}}$, il devient énergétiquement plus favorable de former un réseau de dislocations coins interfaciales. De très nombreuses observations sur des systèmes très variés (métal-métal, métal-semi-

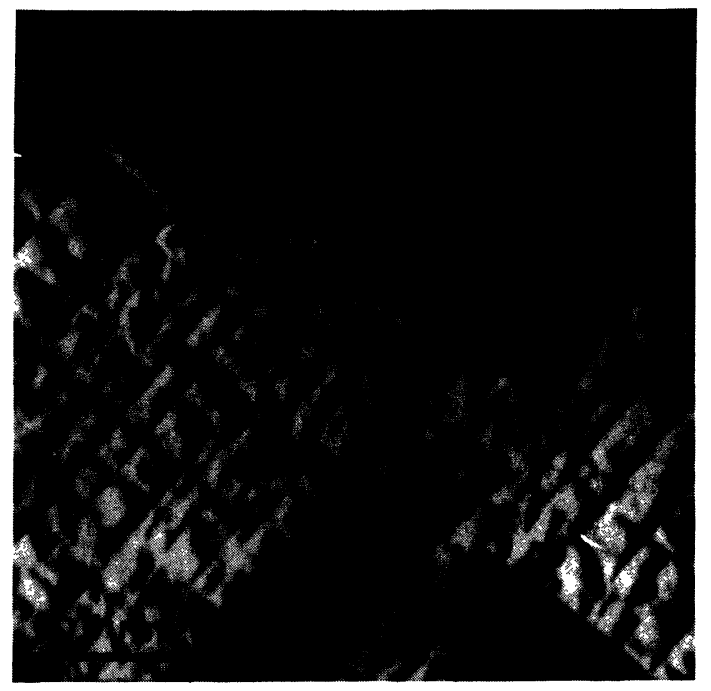

Fig. 1. - Dislocations interfaciales lors de la croissance de palladium (épaisseur $4 \mathrm{~nm}$ ) sur un support d'or orienté (001) d'épaisseur $40 \mathrm{~nm}$.

[Misfit dislocations during the growth of palladium (thickness $4 \mathrm{~nm}$ ) on a gold substrate with an (001) orientation (thickness $40 \mathrm{~nm}$ ).] 
conducteur, semi-conducteur-semi-conducteur...) ont permis de vérifier la validité de ce modèle [11]. Nous en donnons un exemple dans le cas du couple $\mathrm{Pd} / \mathrm{Au}$ (111) étudié par Gillet et coll. [12]. Jusqu'à une épaisseur de dépôt d'environ $1 \mathrm{~nm}$, le dépôt de palladium n'est pas visible ni en microscopie, ni en diffraction électronique. Dans un deuxième stade commençant à una épaisseur de 1 à $2 \mathrm{~nm}$, il apparaît des contrastes alignés dans les directions $\langle 110\rangle$. La figure 2 correspondant à une épaisseur de $4 \mathrm{~nm}$ fait apparaître de longues lignes qui ont été interprétées comme étant des dislocations dont le vecteur de Burgers est de type $\frac{a}{2}\langle 110\rangle$ incliné sur le plan de l'interface; le diagramme de diffraction (Fig. 2) correspondant aux conditions de la figure 1 fait apparaître des points attribuables au dépôt de palladium en supplément de ceux dus au support d'or; la relation d'épitaxie est alors (111) Pd// (111) Au avec [110] Pd 11 [1 $1 \overline{10}] \mathrm{Au} / /$. Aux épaisseurs plus élevées ces micrographies révèlent toujours de longues lignes de dislocations dans les directions $\langle 110\rangle$; ces dislocations ont un vecteur de Burgers $\frac{a}{2}\langle 110\rangle$ incliné dans le plan de l'interface. L'existence de moirés permet de calculer le paramètre du dépôt en supposant que le paramètre du support est celui du métal massif. L'évolution de ce paramètre en fonction de l'épaisseur ne correspond pas aux prévisions théoriques, bien que l'examen en spectroscopie d'électrons Auger de la surface en cours de croissance prouve que cette dernière s'effectue bien couche par couche. Cet écart a été expliqué par Gillet en supposant que les contraintes du dépôt entraînent la formation de dislocations dans le support [13].

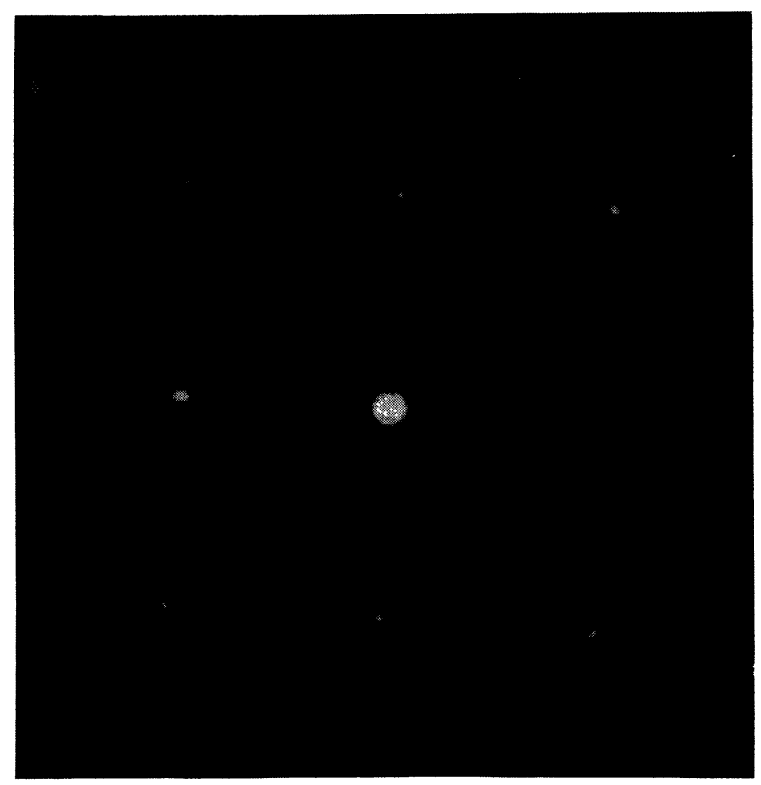

Fig. 2. - Diagramme de microdiffraction correspondant aux conditions de la figure 1 .

[Diffraction pattern corresponding to the conditions of the figure 1.]
Il faut souligner qu'outre la microscopie électronique à transmission, la topographie de Rayons $X$ a été mise en œuvre pour l'étude des dislocations interfaciales et que l'utilisation du rayonnement synchroton devrait connaître maintenant un développement important [14].

4. Défauts d'empilement, macles, double positionnement. - Le mode de croissance des couches minces joue également un rôle important dans la configuration des défauts plans. La microscopie électronique à transmission a permis d'étudier in situ la formation de ces défauts lors de la coalescence d'îlots. On trouvera dans [11], une discussion détaillée de leurs conditions d'apparition et d'observation liées à des déplacements soit par translation, soit par rotation. Ainsi lors de la croissance homoépitaxique, on a fréquemment observé dans les couches minces des tétraèdres de défauts d'empilement. La figure 3 en donne un exemple dans le cas de la croissance épitaxique, par voie électrolytique, du nickel sur un support de cuivre. Booker et Stickler [15] ont montré que ce type de figure était dû à un défaut d'empilement des plans compacts situé entre le support et un germe.

Les possibilités de la microscopie électronique à haute résolution ont conduit récemment à une détermination de l'épaisseur de ces défauts et à distinguer ceux ayant une épaisseur d'une couche atomique (défaut d'empilement intrinsèque), deux couches atomiques (défaut d'empilement extrinsèque) ou plus (micromacles) [16]. La figure 4 tirée de [16] donne l'image d'un dépôt de silicium effectué sur du saphir;

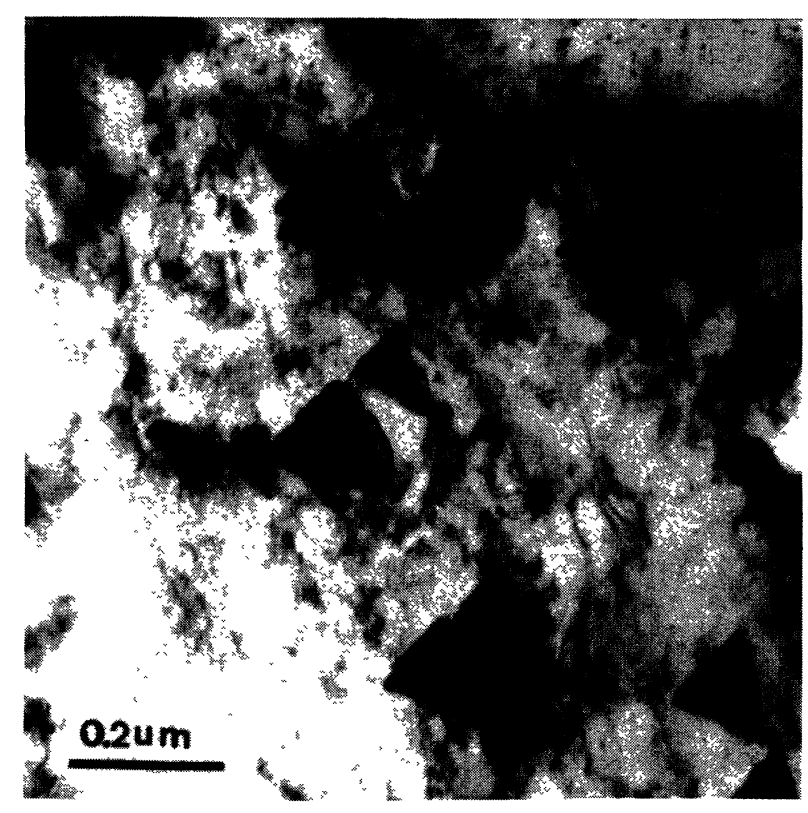

Fig. 3. - Tétraèdres de défauts d'empilements dans un film de nickel épitaxique obtenu par dépôt électrolytique sur un substrat de cuivre (111).

[Stacking fault tetrahedra in an epitaxial film of nickel obtained by electrodeposition on a copper (111) substrate.] 


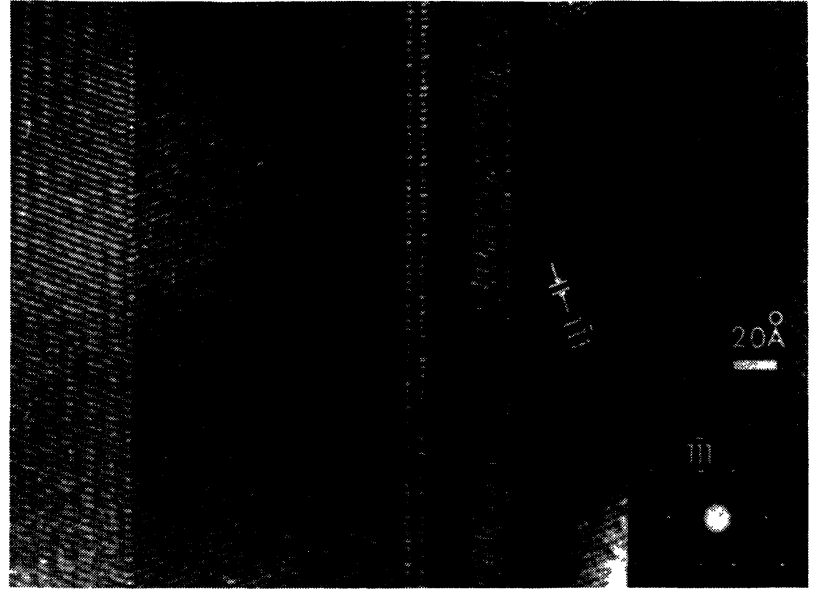

Fig. 4. - Image de réseau d'un film épitaxique de silicium formé sur du saphir (1102); diagramme de diffraction et position du diaphragme d'objectif pour obtenir l'image de réseau.

[Lattice image of a silicon epitaxial film on sapphire (1102); diffraction pattern and position of objective aperture used for lattice image.]

la zone observée contient 4 défauts d'empilement intrinsèques et une micromacle d'une épaisseur de 6 couches atomiques. La figure 5 [16] montre une analyse détaillée des défauts à partir de l'image des plans atomiques. Dans le cas d'une croissance couche par couche, des erreurs d'empilements dans les plans compacts peuvent également se produire. Cela conduit à la formation de défauts d'empilement, de micromacles, diversement inclinées par rapport au plan de la couche mince. Des phénomènes de maclage multiple ont même été observés modifiant ainsi l'orientation générale du dépôt [17]. La figure 6 correspondant à un dépôt électrolytique de nickel effectué sur un support de cuivre d'orientation (100); on observe de nombreuses micromacles inclinées

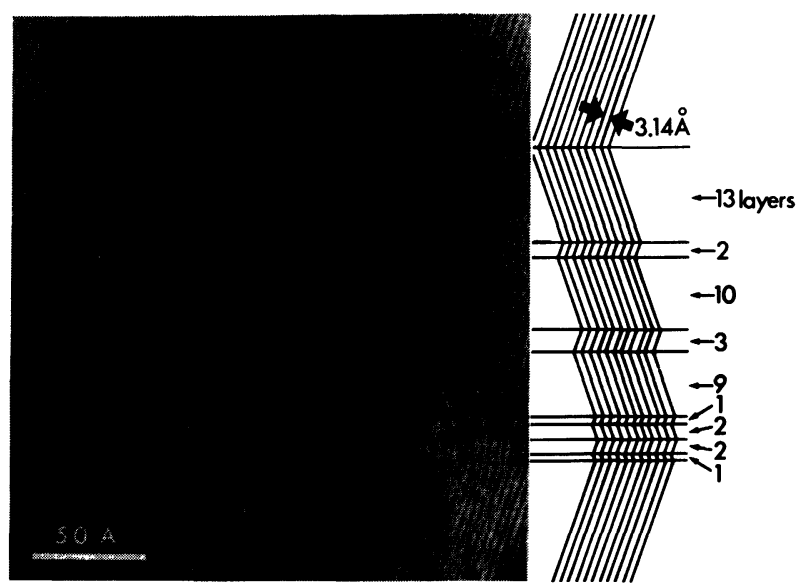

Fig. 5. - Image de réseau d'un film de silicium de $50 \mathrm{~nm}$ d'épaisseur; un schéma des franges de réseau donnant en largeur des micromacles se trouve sur la droite.

[Lattice image of a silicon film (thickness $50 \mathrm{~nm}$ ); the schematic lattice fringes and the thickness of the microtwins observed in the microtwins are illustrated in the right hand side.]

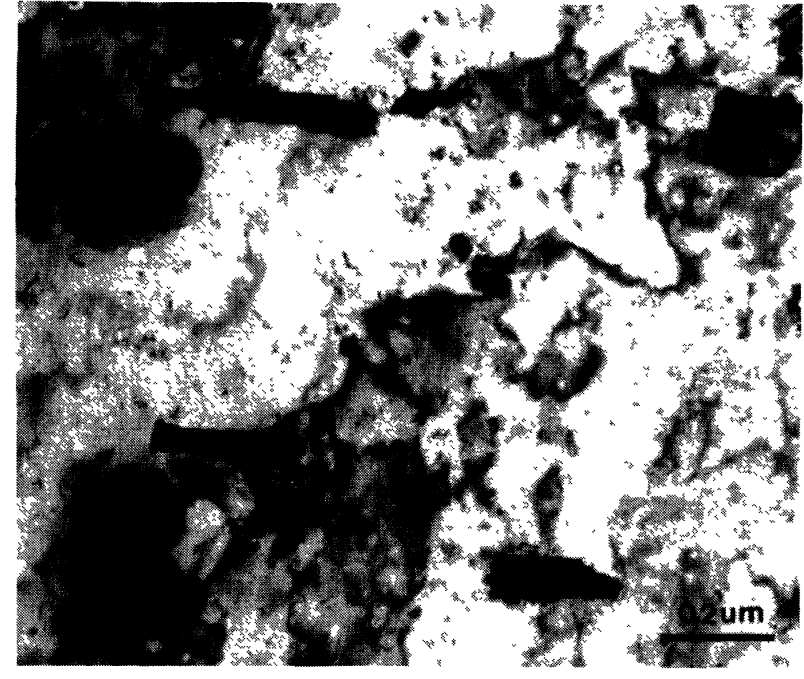

Fig. 6. - Micromacles observées dans un film épitaxique de nickel obtenu par dépôt électrolytique sur un substrat de cuivre (111).

[Microtwins in an epitaxial film of nickel obtained by electrodeposition on a copper (111) substrate.]

dans la couche. Ces défauts, une fois formés, semblent jouer un rôle dans les stades ultérieurs de croissance des couches minces. Plusieurs auteurs ont en effet remarqué que ces micromacles sont à l'origine de figures de croissance de morphologie particulière $[17,18]$. Les erreurs d'empilement sur les plans compacts sont particulièrement importantes lorsque le faciès du dépôt est de type (111). Ainsi B. de Cuminski et Wilman [19] ont observé en diffraction électronique par réflexion la croissance de dépôts électrolytiques de cuivre sur un support monocristallin; la densité de macles est inversement proportionnelle à la vitesse de formation du dépôt ; ceci indique que la densité des macles, liées à la coalescence d'îlots, croît avec la vitesse de croissance. Cependant à une vitesse de croissance donnée, la densité de macles augmente avec l'épaisseur du dépôt. La formation des macles est alors d'autant plus facile que la rugosité du dépôt est plus élevée, c'est-à-dire que la surface contient un plus grand nombre de facettes de type (111). On a également remarqué que les phénomènes d'adsorption, par l'occupation de certains sites du réseau favorisent la formation de macles. Ceci a été fréquemment observé lors du dépôt électrolytique des métaux en présence de composés organiques susceptibles de fortes interactions avec la surface [20]. L'examen de lames minces a ainsi révélé une augmentation de la densité de macles et de défauts d'empilement après addition de saccharine à des solutions de dépôt électrolytique de nickel [21]. Enfin la coalescence de 2 îlots où l'empilement est respectivement de type Abcabc... et Acbacb... (A représentant la position des atomes dans le plan du substrat) conduit à la formation de parois de double positionnement [11]. Ces parois d'énergie élevée sont souvent séparées par des marches parallèles au substrat 
alternant ainsi des plans (111) et (211) dans le cas où le substrat est constitué par un plan (111).

5. Edifices multimaclés. - La formation de défauts plans telle que nous l'avons décrite jusqu'ici résultait de phénomènes post nucléatoires liés à la coalescence d'îlots ou à des erreurs d'empilements dans les plans compacts. On observe également des arrangements très particuliers de macles dont l'origine remonte au phénomène de nucléation lui-même. En général, les édifices multimaclés sont observés lorsque la croissance a lieu par îlots tridimensionnels avec de faibles interactions entre le dépôt et le support $\left(\psi_{\mathrm{AB}}<\psi_{\mathrm{AA}}\right)$ [2]. De nombreux travaux théoriques ont montré que l'arrangement stable d'agrégats contenant un très petit nombre d'atomes de métaux c.f.c. était caractérisé par l'apparition de symétries pentagonales [22, 23, 24]. Nous ne discuterons pas ce point qui fait l'objet d'un colloque tenu lors de ce congrès. Nous soulignerons cependant le rôle essentiel joué par les observations très fines, en microscopie électronique, de nombreux expérimentateurs qui ont conduit à interpréter les anomalies de contraste et les diagrammes de diffraction présentés par ces agrégats. Dans la plupart des cas, les observations ont concerné les tous premiers stades de nucléation et de croissance de germes d'or évaporés sur des supports de $\mathrm{KCl}$ ou de $\mathrm{NaCl}[25,26,27,28]$. Nous insisterons également sur les conséquences de la formation de tels germes, lors des stades ultérieurs de la croissance. En effet, les agrégats présentant des symétries pentagonales ne peuvent pas croître indéfiniment avec de fortes distorsions dans les distances inter-réticulaires par rapport à celles présentées par la structure c.f.c. Des observations en microscopie électronique à haute résolution de petits germes d'or ont montré qu'au-delà d'une certaine taille (environ $100 \AA$ ) [29], ces germes paracristallins dégénèrent en édifices multimaclés; dans ces édifices la symétrie pentagonale est conservée par l'arrangement des plans de macle. En effet l'angle entre 2 plans de macle (111) est inférieur à $2 \pi / 5$ et il subsiste dans l'édifice un défaut angulaire de $7^{\circ} 20^{\prime}$. La proportion de germes paracristallins puis d'édifices multimaclés reste en général assez faible lors de la croissance à partir de la phase vapeur [30]. Par contre, ce phénomène peut prendre une grande importance lors de l'électrocristallisation de métaux c.f.c. comme le nickel [31] et l'argent [32]. On peut penser que la proportion importante de germes paracristallins est alors liée aux sursaturations élevées qui règnent à l'interface lors de l'électrocristallisation. Par ailleurs, les phénomènes d'inhibition qui accompagnent en particulier l'électrocristallisation du nickel jouent probablement un rôle dans la stabilisation des germes paracristallins [33]. Enfin des phénomènes de sélection dans les premiers instants de la croissance contribuent aussi à renforcer la proportion d'édifices multimaclés [34]. D'autres arrangements de plans de macles que ceux présentant une symétrie pentagonale ont été aussi observés; ils dérivent probablement de germes paracristallins. Ainsi les observations de dépôts électrolytiques de nickel présentant une texture de fibre d'axe [211] révèlent une grande proportion de grains contenant des macles rapprochées (Fig. 7a). Des observations de lames minces monocristallines de nickel sur lesquelles ont été effectuées des dépôts de très faible épaisseur ont montré la formation de germes dont on peut penser qu'ils dérivent d'un édifice isocaédrique comme le montre la figure $7 b$ [35]. Les défauts de fermeture dus aux arrangements pentagonaux d'atomes entraîneraient, lors de la croissance, la formation de plans de macles rapprochés. Il faut noter que ces arrangements de plans de macle parallèles ou en étoile, issus d'agrégats paracristallins, jouent un rôle très actif dans la croissance orientée
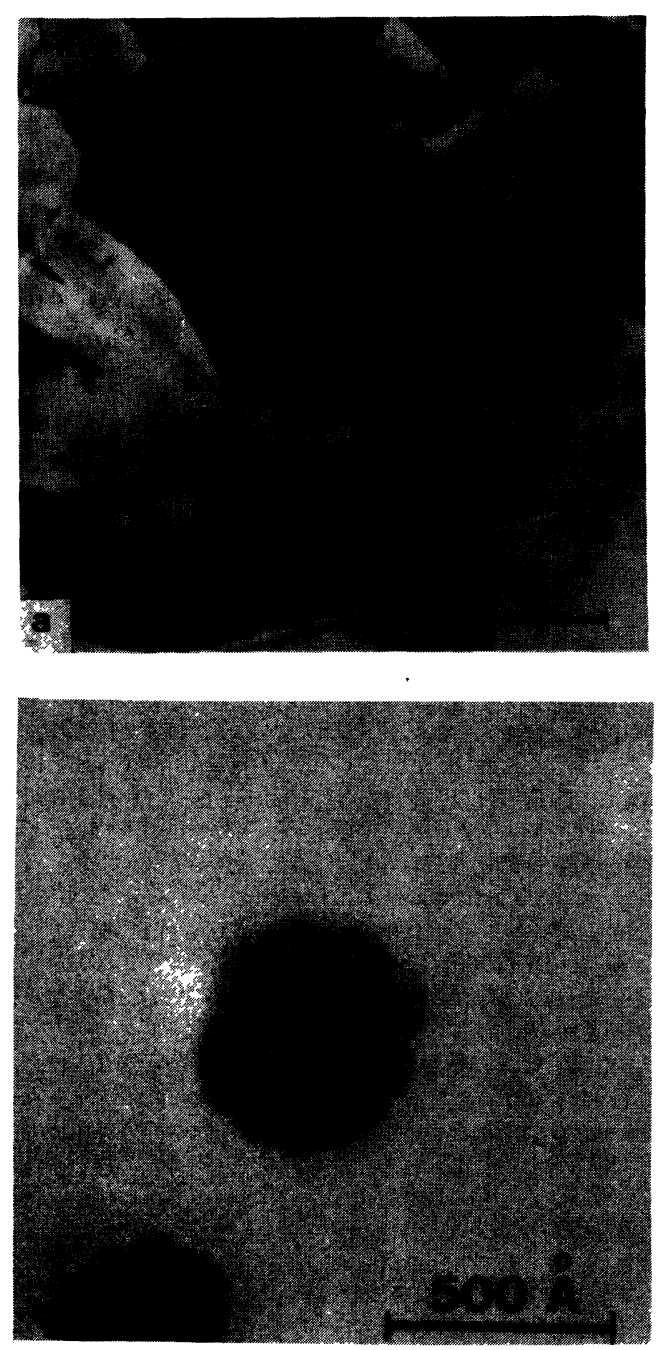

Fig. 7. - Dépôts électrolytiques de nickel : $a$ ) lame mince obtenue à partir d'un dépôt épais présentant un axe de texture $\langle 211\rangle$; b) cristallite multimaclé obtenu sur un substrat mince de nickel monocristallin; microscopie électronique à haute tension.

[Nickel electrodeposits : $a$ ) thin foil obtained from a thick electrodeposit with a $\langle 211\rangle$ texture axis; $b$ ) multitwinned crystallite obtained on a thin nickel single crystal; high tension electron microscopy.] 
des dépôts. En effet, l'association de plans de macles rapprochés et de faciès (111) entretient la présence d'angles rentrants qui sont des sites actifs pour l'incorporation des atomes [36]. Il en résulte une croissance accélérée dans des directions [211] ou [110], à l'origine de la formation de dépôts texturés et du déclenchement de la croissance dendritique [32]. Dans le cas des plans de macle groupés en étoile, on peut penser que l'arrangement des atomes au point de concours provoque l'existence d'un gradin engendrant, comme dans le cas d'une dislocation vis, une croissance hélicoïdale continue [37].

Enfin nous signalerons l'observation de défauts très particuliers dont on peut penser qu'ils ne sont pas dus à des phénomènes aléatoires de coalescence mais qu'ils trouvent leur origine dès le stade de nucléation. Ainsi lors de l'électrocristallisation du cobalt [38] et du plomb [39] et dans les dépôts de cadmium [40], on a mis en évidence des défauts plans analogues à des joints de torsion. Ces défauts offrent sur leur pourtour de nombreux sites d'incorporation facile des atomes qui sont à l'origine d'une croissance accélérée dans les directions contenues dans le plan du joint de torsion.

6. Conclusion. - Après ce rapide survol où nous nous sommes efforcés de montrer quelques possibilités de la microscopie électronique dans le très vaste domaine de la formation des défauts au cours de la croissance de divers matériaux en couches minces, il est opportun de se demander si dans les années à venir, un effort aussi important sera maintenu et quelle sera la place tenue par la microscopie électronique. Les problèmes liés aux sources et convertisseurs d'énergie (solaire, électrochimique, ...) d'une part, le développement de dispositifs de plus en plus perfectionnés formés de structures multicouches, exigés par l'évolution actuelle des composants pour l'électronique et l'informatique d'autre part, vont sans aucun doute jouer un rôle moteur. Ce dernier domaine exige en effet la préparation de couches constituées de matériaux (métaux, semi-conducteurs, oxydes, ...) dont l'épitaxie, l'épaisseur, la composition, la densité des défauts sont parfaitement contrôlées [41]. Il est clair que grâce aux améliorations apportées progressivement, tant en ce qui concerne la résolution, l'interprétation des contrastes, les possibilités d'une analyse chimique localisée, la microscopie électronique, restera une méthode de choix pour l'étude des couches minces. Il n'en est pas moins évident que des méthodes comme la topographie de Rayons X (rayonnement synchrotron), et les diverses spectroscopies d'électrons ou d'ions confirmeront la place de plus en plus importante qu'elles ont prises ces dernières années.

Remerciements. - L'auteur est heureux de remercier MM. M. Gillet, T. Hayashi, S. Kurosawa et G. Maurin pour les clichés qu'ils ont bien voulu lui confier.

\section{Bibliographie}

[1] Bauer, E., Z. Phys. 110 (1958) 372

[2] Le Lay, G., Manneville, M., Kern, R., Surf. Sci. 72 (1978) 405.

[3] Sato, M., Shinozaki, S., Surf. Sci. 22 (1970) 229.

[4] Le Lay, G., Kern, R., J. Cryst. Growth 44 (1978) 197.

[5] Budevski, E. B., Prog. Surf. Membr. Sci. 11 (1976) 116.

[6] Froment, M., Maurin, G., Thevenin, J., Mét.-Corros., no 536 (1970) 123.

[7] Bebczuk de Cuminsky, J., J. Cryst. Growth 41 (1977) 330.

[8] Jacobs, M. N., Pashley, D. W., Stowell, M. J., Philos. Mag. 13 (1966) 129.

[9] Masson, A., Metois, J. J., Kern, R., Surf. Sci. 27 (1971) 463 et 483.

[10] Frank, F. C., van der Merwe, J. H., Proc. R. Soc. A 198 (1949) 205.

[11] Epitaxial Growth, Edité par J. W. Matthews (Academic Press) 1975.

[12] Hila-Slim, A., Gillet, M., J. Microsc. Spectrosc. Electron. 2 (1977) 223.

[13] Bruzza, B., Guglilemacci, J. M., Gillet, E., Thin Solid Films 52 (1978) 103.

[14] Petroff, J. F., Sauvage, M., J. Cryst. Growth 43 (1978) 628.

[15] Booker, G. R., Stickler, R., J. Appl. Phys. 33 (1962) 3281.

[16] Hayashi, T., Kurosawa, S., J. Cryst. Growth 45 (1978) 426.

[17] Fraise, P., Froment, M., Laupretre, J. M., Maurin, G., J. Microsc. 17 (1973) 1.

[18] SARD, R., WeIL, R., Electrochim. Acta 15 (1970) 1977.

[19] Bebzux de Cuminsky, J., Wilman, M., Electrochim. Acta 17 (1972) 237.

[20] Mamontov, E. A., Kozlov, V. M., Kurbatova, L. A., Sov Electrochem. 13 (1977) 142.
[21] Froment, M., J. Microsc. 3 (1964) 61.

[22] Hoare, M. R., Pal, P., J. Cryst. Growth 17 (1972) 77.

[23] Julg, A., Bourg, M., Surf. Sci. 34 (1972) 709.

[24] Leleyter, M., Joyes, P., J. Physique Lett. 37 (1976) L-303.

[25] Ino, S., J. Phys. Soc. Japan 21 (1966) 346.

[26] Allpress, J. G., Sanders, J. V., Surf. Sci. 7 (1967) 1.

[27] Komoda, T., Japan. J. Appl. Phys. 7 (1968) 27.

[28] Gillet, E., Gillet, M., Thin Solid Films 4 (1969) 1971

[29] Gillet, E., Gillet, M., Thin Solid Films 29 (1975) 217.

[30] Renou, A., Gillet, M., Thin Solid Films 41 (1977) 15.

[31] Froment, M., Maurin, G., J. Microsc. 7 (1968) 39.

[32] Digard, C., Maurin, G., Robert, J., Mét. Corros. 611-612 (1976) 255-263.

[33] Amblard, J., Epelboin, I., Froment, M., Maurin, G., J. Appl. Electrochem. 9 (1979) 233.

[34] Maurin, G., Conf. 32e Réunion de la Soc. Chim. Physique, Villeurbanne, Septembre 1979 ; Volume édité par Elsevier, sous presse.

[35] Thevenin, J., J. Microsc. Spectrosc. Electron. 1 (1976) 7.

[36] Hamilton, D. R., Seidensticker, R. G. J., Appl. Phys. 31 (1960) 1165.

[37] Melmed, A. J., Hayward, P. O., J. Chem. Phys. 31 (1959) 541.

[38] Epelboin, I., Froment, M., Maurin, G., Plating 12 (1969) 1356.

[39] Maurin, G., Mercier, D., C. R. Hebd. Séan. Acad. Sci. B 282 (1976) 99.

[40] Price, P. B., Philos. Mag. 4 (1959) 1229.

[41] Acta Electron. 21 (1978) n n $^{\text {s }} 2$ et 3. 